\title{
New Physics search in rare $\tau$ decays at Belle and prospects at Belle II
}

\author{
Nobuhiro Shimizu for the Belle collaboration*† \\ University of Tokyo, Aihara/Yokoyama laboratory, \\ Department of Physics, \\ 7-3-1 Hongo, Bunkyo-ku, Tokyo 113-0033 JAPAN \\ E-mail: shimizu@hep.phys.s.u-tokyo.ac.jp
}

\begin{abstract}
We present our recent achievements and ongoing activities about the search for the New Physics using rare decay of $\tau$. The rare decays are categorized into two groups. First one encompasses practically forbidden decays, in particular, the Lepton flavor violating decays are reviewed. The other group includes tau decays with suppressed branching fractions, predicted by the Standard Model. Both of them provide good opportunities to find hints of the New Physics.
\end{abstract}

Flavor Physics and CP Violation

6-9 June 2016

Caltech, Pasadena CA, USA

\footnotetext{
*Speaker.

${ }^{\dagger}$ Belle collaboration.
} 


\section{Introduction}

The decay of tau is known as one of the most important tools to search for New Physics (NP). The large mass of $\tau\left(m_{\tau}=1776.86 \pm 0.12 \mathrm{MeV}\right.$ [1]) allows us to expect an enhancement of the sensitivity to the NP. For instance, the NP models like 2HDM (two Higgs doublet model) predict existence of the charged Higgs and the magnitude of their couplings are proportional to mass of leptons. Therefore, in comparison with muon decays, we can expect the gain of a factor $\left(m_{\tau} / m_{\mu}\right)^{2} \sim 300$. Moreover, it is even possible to consider so called heavy stable particles in the final state.

The mass of $\tau$ makes it possible to decay into both leptons and hadrons. The former one is called leptonic decay and accounts for approximately 35\% of all tau decays. Various properties of these decays, described by the electroweak sector of the SM, are precisely calculated, hence experimental results can be definitely compared with theoretical predictions. The rest decays of tau contain hadrons in the final state and called hadronic decay. These decays turn out to be beautiful laboratory for studying low energy structure of the strong interaction like the chiral perturbation theory.

Among many strategies for the NP discovery in tau decays, the studies of the rare decays are useful approaches. These decays are categorized into two groups: one is forbidden and the other is suppressed ones. Both of them can be reinterpreted that the SM backgrounds are limited, hence they are effectively sensitive to the NP phenomena.

One of the example of the first category is lepton flavor violating (LFV) decay modes. In the standard model (SM), each lepton flavor $N_{e}, N_{\mu}$ and $N_{\tau}$ is separately conserved. Therefore, the decay of tau always entails tau neutrino - only known particle which has tau flavor and smaller mass than tau lepton. LFV decays do not contain neutrinos in the final state and it is experimentally possible to observe all outgoing particles. The characteristic feature of these LFV decays are utilized to search for them. The other category is suppressed decay.

To proceed to the study of these rare decays, huge number of $\tau$ is necessary where $\sim \mathrm{O}\left(10^{8}\right)$ of $\tau$ is required. The KEKB electron positron accelerator was originally developed for the production of $B$ mesons, so called $B$ factory, where the world largest instantaneous luminosity of $2 \times 10^{34} \mathrm{~cm}^{2} \mathrm{~s}^{-1}$ was reached. It is, however, also known as $\tau$ factory. The clean environment the Belle detector enables us to perform detailed analysis of tau leptons as well.

\section{Belle and Belle II experiment}

The Belle experiment at KEK (Tsukuba, Japan) collected $1040 \mathrm{fb}^{-1}$ of data sample using $e^{+} e^{-}$energy-asymmetric collider KEKB. It ran from June 1999 to June 2010 and contributed to the progress of high energy physics, most notably a verification of a mechanism of $\mathrm{CP}$ violation which resulted in the Nobel prize in 2008. Figure 1 shows the overall view of the Belle detector. It is designed to be multi-purpose so that vertexing, momentum tracking, energy measurement of photon and particle identification are interactively performed by several sub-detectors. Although Belle experiment achieved many findings, nevertheless there are some possible hints of the NP which need to be confirmed with more precise measurements. 
Faced with the situation, Belle II experiment is being planned to start to start data taking with $e^{+} e^{-}$energy-asymmetric collider SuperKEKB from 2017. With the upgrade of accelerator, the luminosity, $80 \times 10^{34} \mathrm{~cm}^{-2} \mathrm{~s}^{-1}$, becomes 40 times as large as that of former experiment Belle. It is expected that the sign of new physics will be found with the largest statistics the world never seen. Since the upgrade of accelerator requires detector also to improve its performance, upgrade of the detector for Belle II is ongoing.

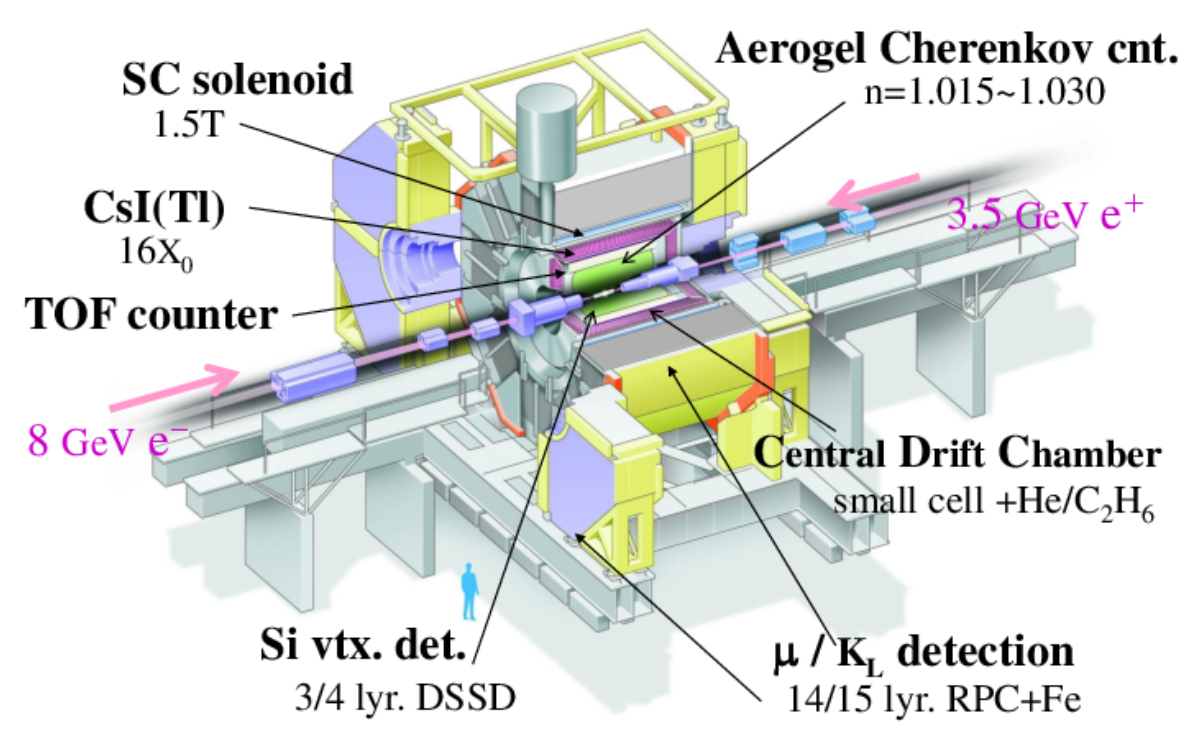

Figure 1: Overall view of Belle detector. The Belle detector is comprised of several sub-detectors. The details can be seen in Ref. [2].

These $B$ factory experiments are useful laboratory also for the study of the $\tau$ leptons. At the resonance energy of $\Upsilon(4 S) \sim 10.58 \mathrm{GeV}$, the cross section of tau pair production is compatible with that of $b b$ pair $\left(\sigma_{e e \rightarrow \tau \tau}=0.9 \mathrm{nb}^{-1}\right.$ and $\left.\sigma_{e e \rightarrow b b}=1.1 \mathrm{nb}^{-1}\right)$. Therefore, sometimes $B$ factory is called $\tau$ factory where KEKB accelerator produced $\mathrm{O}\left(10^{9}\right) \tau \tau$ pairs in total. Moreover, the clean environment of e+e- collider enables us to reconstruct all outgoing long-living particles (except neutrinos) with high purity, which opens unique opportunities of many precision studies of tau leptons.

\section{Search for LFV decay}

In the SM, the conservation of all lepton numbers $\left(N_{e}, N_{\mu}\right.$ and $\left.N_{\tau}\right)$ exactly hold in every interaction. Therefore, the discovery of the LFV decay immediately suggests the existence of the NP. The only known phenomena of the LFV process is the neutrino oscillation and the observed $\Delta m_{v}^{2}$ implies the branching ratio of tau decay $\mathscr{B}$ up to order of $10^{-54}[3]$ [4]. On the other hand, many NP models predict the enhancement of the branching ratio of LFV decay up to order of $\sim 10^{-8}$. In Table 1, the impacts of the NP models are summarized for $\tau \rightarrow \mu \gamma$ and $\tau \rightarrow \mu \mu \mu$ cases. 
Table 1: Possible enhancement of branching ratio of LFV decay modes

\begin{tabular}{lccr}
\hline \hline NP Model & $\mathscr{B}(\tau \rightarrow \mu \gamma)$ & $\mathscr{B}(\tau \rightarrow \mu \mu \mu)$ & Ref. \\
\hline SM+heavy Majorana $v_{R}$ & $10^{-9}$ & $10^{-10}$ & $\square 5$ \\
Non-universal $Z^{\prime}$ & $10^{-9}$ & $10^{-8}$ & $\square[$ \\
SUSY SO $(10)$ & $10^{-8}$ & $10^{-10}$ & $\square]$ \\
mSUGRA+seesaw & $10^{-7}$ & $10^{-9}$ & $\square]$ \\
SUSY Higgs & $10^{-10}$ & $10^{-7}$ & $\square 9$ \\
\hline \hline
\end{tabular}

The conservation of tau lepton number leads that the decay of $\tau$ must always occur with tauneutrino. For instance, the leptonic and hadronic decay respectively contain two and one neutrinos in the final state. On the other hand, if LFV decay occurs no neutrinos appear, hence all outgoing particles can be experimentally observed. This nature allows us to reconstruct mass of tau from all observed four-vectors and suppress sources of the SM backgrounds with retaining reasonable efficiency.

\section{1 $\tau \rightarrow l \gamma$ and $\tau \rightarrow l l l(l=e$ or $\mu)$}

The analysis of these decays are performed on two-dimension plane $\left(m_{\mathrm{obs}}, \Delta E\right)$ where $m_{\mathrm{obs}}$. is the invariant mass of all observed particles and $\Delta E$ is the energy loss defined as $E_{\text {beam }}-E_{\text {obs. }}$ In the case of signal, these variables show peak structure around $\left(m_{\text {obs. }}, \Delta E\right)=\left(m_{\tau}, 0\right)$ and enables us to reject contamination of backgrounds by applying elliptical signal window.

Belle experiment searched for $\tau \rightarrow l \gamma$ and $\tau \rightarrow l l l$ decays using $535 \mathrm{fb}^{-1}$ and $782 \mathrm{fb}^{-1} \mathrm{ac}^{-}$ cumulated data respectively [10] [11]. Figure 2 and Fig. 3] show plots of $\left(m_{\text {obs. }}, \Delta E\right)$ for observed candidates and these corresponding results are summarized in Table $2 \tau \rightarrow l \gamma$ decay modes are suffered from contamination of backgrounds than $\tau \rightarrow l l l$ case. For both cases, major background contributions are radiative Bhabha (ee $\rightarrow l l \gamma)$ and initial state radiation (ISR) $(e e \rightarrow \tau \tau \gamma)$.

To maintain the linearity of the sensitivity of these decays with the integrated luminosity at Belle II , it is important to reject beam background, which is not proportional to the luminosity. So far, the fraction of this background was approximately ten percent of ISR processes, but it is a key how to tolerate 40 times larger luminosity. With the upgrade of electromagnetic calorimeter of Belle II detector, newly developed waveform sampling of photon clustering will be adopted and the hit time detection enables us to suppress the accidental beam background. Moreover, the trigger system will be upgraded where more dedicated separation of $\tau \rightarrow l \gamma$ and Bhabha events will be performed. Figure 4 shows the expected limit of the sensitivity for future integrated luminosity.

\subsection{Other LFV decays}

Not only did we search for $\tau \rightarrow l \gamma$ and $\tau \rightarrow l l l$ decays but we also studied other LFV decay modes in around fifty possible decay modes as illustrated in Fig 5 Some of them even break other conservations like the lepton number and baryon number as typified by $\tau^{-} \rightarrow \mu^{+} e^{-} e^{-}$and $\tau \rightarrow \lambda \pi$ decays respectively. The general observation of this figure is that obtained upper limits are of the order of $\mathrm{O}\left(10^{-8}-10^{-7}\right)$ and provide notable constraints of various NP-models. Belle II is expected 


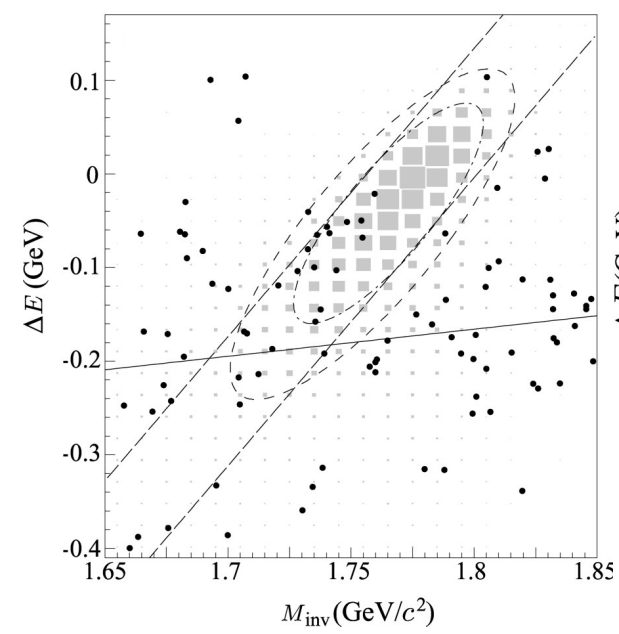

(a) $\tau \rightarrow e \gamma$

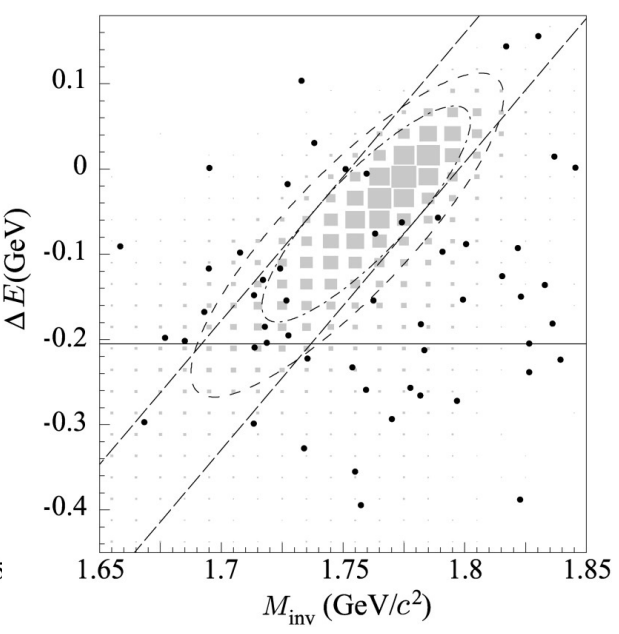

(b) $\tau \rightarrow \mu \gamma$

Figure 2: Distribution of $\left(m_{l \gamma}, \Delta E\right)$ for obtained candidates of (a) $\tau \rightarrow e \gamma$ and (b) $\tau \rightarrow \mu \gamma$. Dotdashed line represents the $2 \sigma$ signal region and dashed one is $3 \sigma$ blinded region and points show experimental data.

Table 2: Obtained upper limit (90\% confidence level)

\begin{tabular}{lcccc}
\hline \hline Mode & $N_{\text {obs. }}$ & $\varepsilon(\%)$ & Upper limit $\left(10^{-8}\right)$ & Note \\
\hline$\tau \rightarrow e \gamma$ & 10 & 3.0 & 12 & \\
$\tau \rightarrow \mu \gamma$ & 5 & 5.1 & 4.5 & \\
$\tau \rightarrow e e e$ & 0 & 6.0 & 2.7 & \\
$\tau \rightarrow \mu \mu \mu$ & 0 & 7.6 & 2.1 & \\
$\tau^{-} \rightarrow e^{-} \mu^{+} \mu^{-}$ & 0 & 6.1 & 2.7 & \\
$\tau^{-} \rightarrow \mu^{-} e^{+} e^{-}$ & 0 & 9.3 & 1.8 & \\
$\tau^{-} \rightarrow \mu^{-} e^{+} \mu^{-}$ & 0 & 10.1 & 1.7 & LFV\& LNV \\
$\tau^{-} \rightarrow e^{-} \mu^{+} e^{-}$ & 0 & 11.5 & 1.5 & LFV\& LNV \\
\hline \hline
\end{tabular}

to reach better sensitivity, which will result in the discovery of LFV decay or exclusion of many NP-models in the wider parametric domain. Furthermore, as shown in Table 3, even once LFV decays are observed, it is required to search for multiple LFV decay modes in order to discriminate many NP models because every theory predicts different branching ratio.

As more general approach for the LFV processes, the analysis of $\tau \rightarrow l X(l=e$ or $\mu)$ decay is ongoing, where $X$ represents an invisible particle which goes through detectors. This measurement was first reported by ARGUS experiment [14]. The key signature of this decay is extraction of the momentum of lepton inside tau rest frame. As shown in Fig 6 , the spectrum of the momentum of lepton turn out to be the delta-like distribution only when the invisible particle $X$ exists. The spread of this distribution (hence equivalent to expected signal to noise ratio) depends on the precision of the determination of tau direction. The ARGUS experiment used heavy hadronic decays $\tau \rightarrow$ $\pi \pi \pi\left(\pi^{0}\right)$ on the other side of tau and approximated the direction of signal side of tau as reversal 

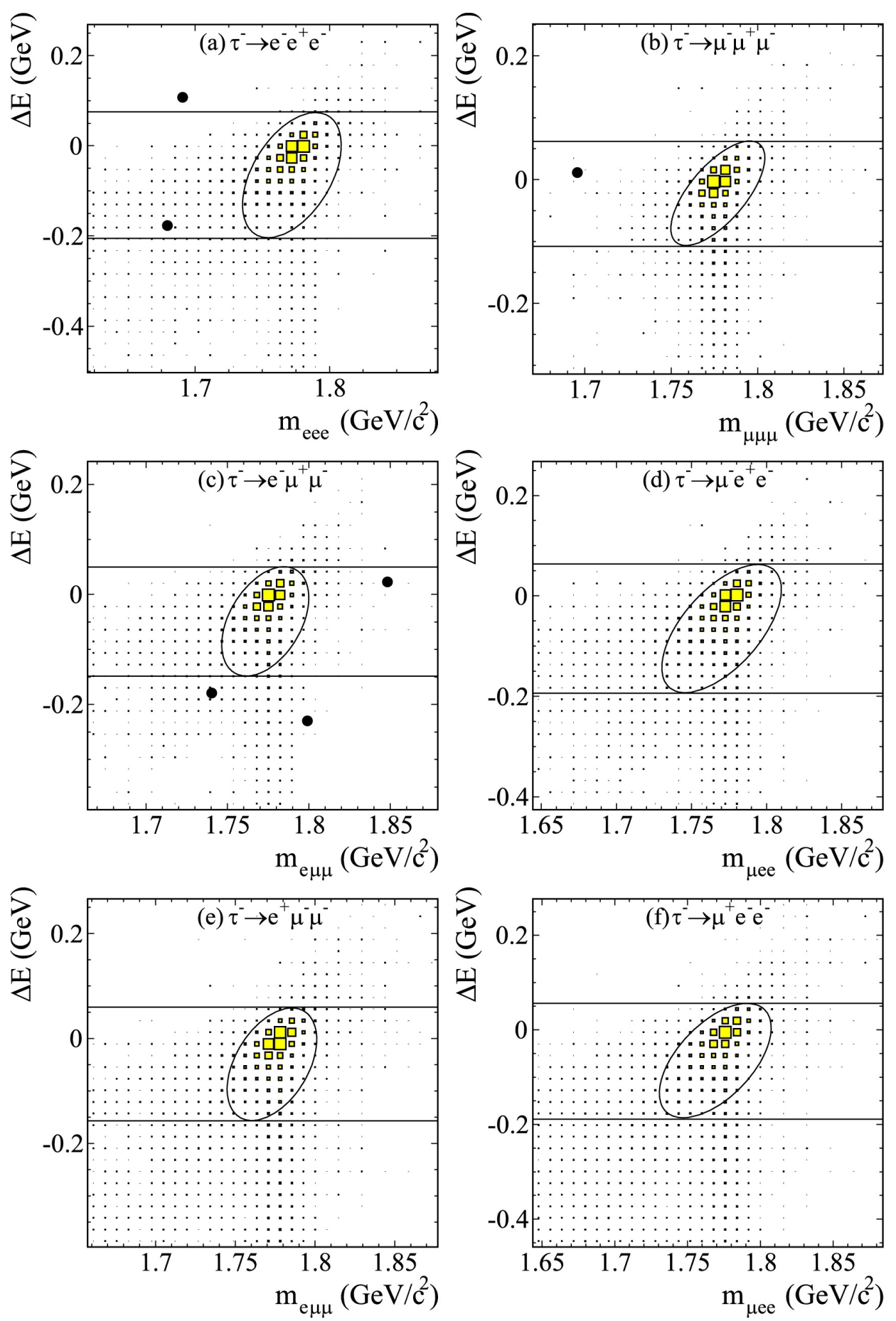

Figure 3: Distribution of $\left(m_{l l l}, \Delta E\right)$ for obtained candidates of (a) $\tau \rightarrow e e e$, (b) $\tau \rightarrow \mu \mu \mu$, (c) $\tau^{-} \rightarrow e^{-} \mu^{+} \mu^{-}$, (d) $\tau^{-} \rightarrow \mu^{-} e^{+} e^{-}$, (e) $\tau^{-} \rightarrow \mu^{-} e^{+} \mu^{-}$and (f) $\tau^{-} \rightarrow e^{-} \mu^{+} e^{-}$. The elliptical lines represent the $2 \sigma$ signal regions and yellow filled boxes show signal MC distribution in arbitrary units. Black points show experimental data. 


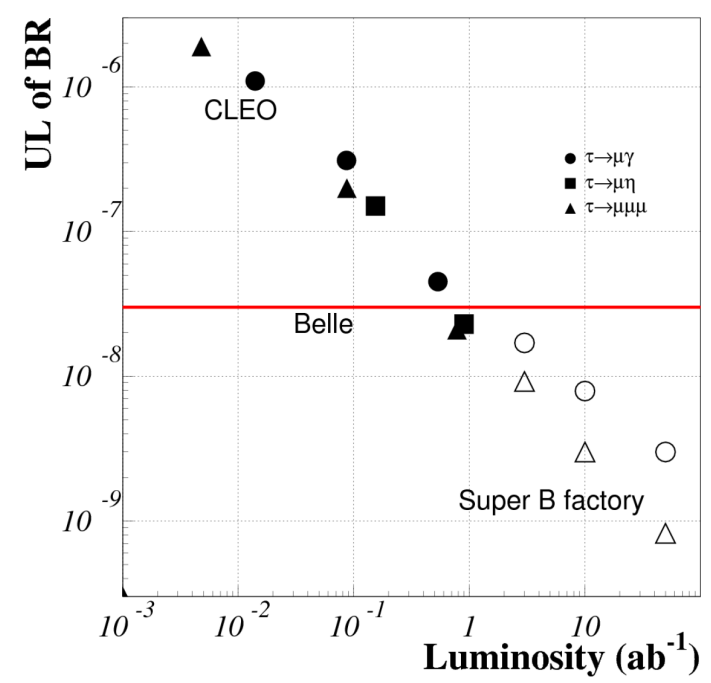

Figure 4: Expected upper limits of LFV decay modes as a function of the integrated luminosity.

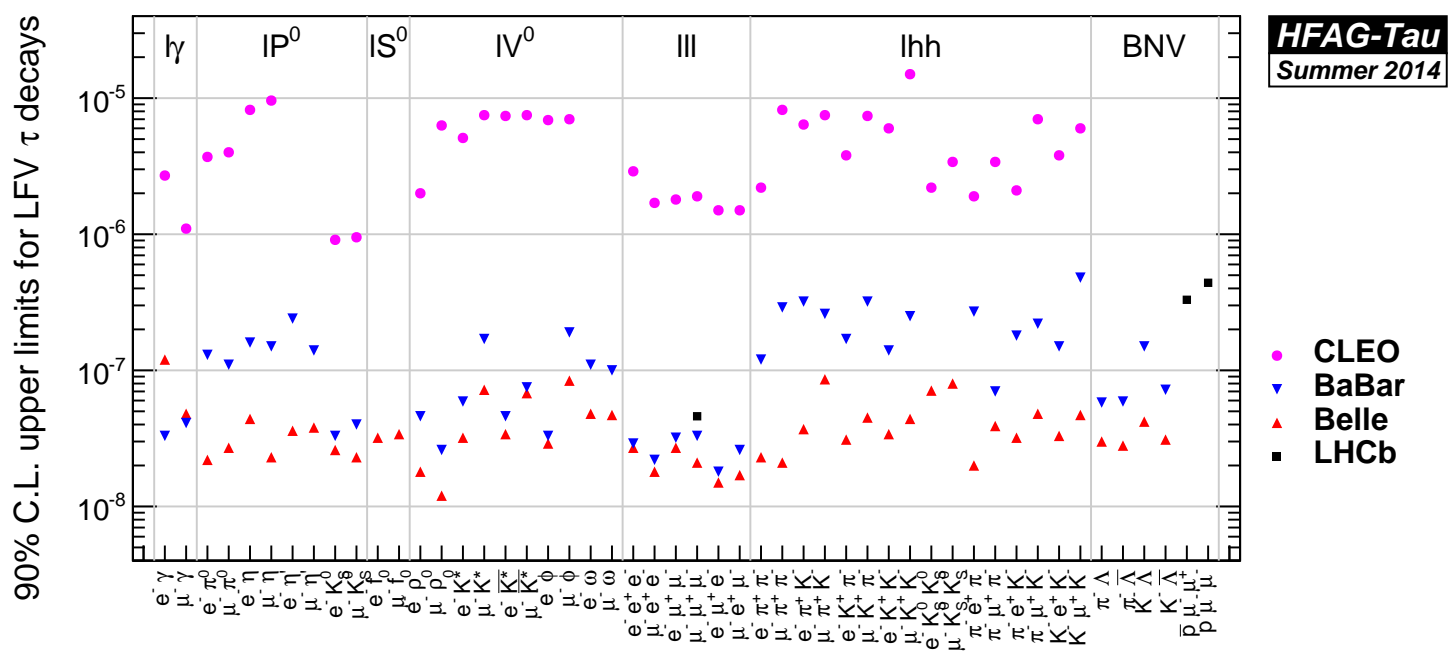

Figure 5: Obtained upper limits (90\% confidence level) for various LFV decay modes [12].

way of the hadrons. With this technique, ARGUS obtained upper limit of ratio of these decays as $\mathscr{B}(\tau \rightarrow e X) / \mathscr{B}(\tau \rightarrow e v \bar{v})<1.5 \%$ and $\mathscr{B}(\tau \rightarrow \mu X) / \mathscr{B}(\tau \rightarrow \mu \nu \bar{v})<2.6 \%$ at $95 \%$ confidence level. 
Table 3: Ratios of the branching fractions predicted by various NP-models [13]

\begin{tabular}{l|ccc}
\hline \hline Ratio & Littlest Higgs with T-parity & MSSM (dipole dominant) & MSSM (Higgs significant) \\
\hline$\frac{\mathscr{B}(\mu \rightarrow e e e)}{\mathscr{B}(\mu \rightarrow e \gamma)}$ & $0.4-2.5$ & $\sim 6 \cdot 10^{-3}$ & $\sim 6 \cdot 10^{-3}$ \\
$\frac{\mathscr{B}(\tau \rightarrow e e)}{\mathscr{B}(\tau \rightarrow e \gamma)}$ & $0.4-2.3$ & $\sim 1 \cdot 10^{-2}$ & $\sim 1 \cdot 10^{-2}$ \\
$\frac{\mathscr{B}(\tau \rightarrow \mu \mu \mu)}{\mathscr{B}(\tau \rightarrow \mu \gamma)}$ & $0.4-2.3$ & $\sim 2 \cdot 10^{-3}$ & $0.06-0.1$ \\
$\frac{\mathscr{B}(\tau \rightarrow e \mu \mu)}{\mathscr{B}(\tau \rightarrow e \gamma)}$ & $0.3-1.6$ & $\sim 2 \cdot 10^{-3}$ & $0.02-0.04$ \\
$\frac{\mathscr{B}(\tau \rightarrow \mu e)}{\mathscr{B}(\tau \rightarrow \mu \gamma)}$ & $0.3-1.6$ & $\sim 1 \cdot 10^{-2}$ & $\sim 1 \cdot 10^{-2}$ \\
$\frac{\mathscr{B}(\tau \rightarrow e e e}{\mathscr{B}(\tau \rightarrow e \mu \mu)}$ & $1.3-1.7$ & $\sim 5$ & $0.3-0.5$ \\
$\frac{\mathscr{B}(\tau \rightarrow \mu \mu \mu)}{\mathscr{B}(\tau \rightarrow \mu e e)}$ & $1.2-1.6$ & $\sim 0.2$ & $5-10$ \\
$\frac{R(\mu \mathrm{Ti} \rightarrow e \mathrm{Ti})}{\mathscr{B}(\mu \rightarrow e \gamma)}$ & $10^{-2}-10^{2}$ & $\sim 5 \cdot 10^{-3}$ & $0.08-0.15$ \\
\hline \hline
\end{tabular}

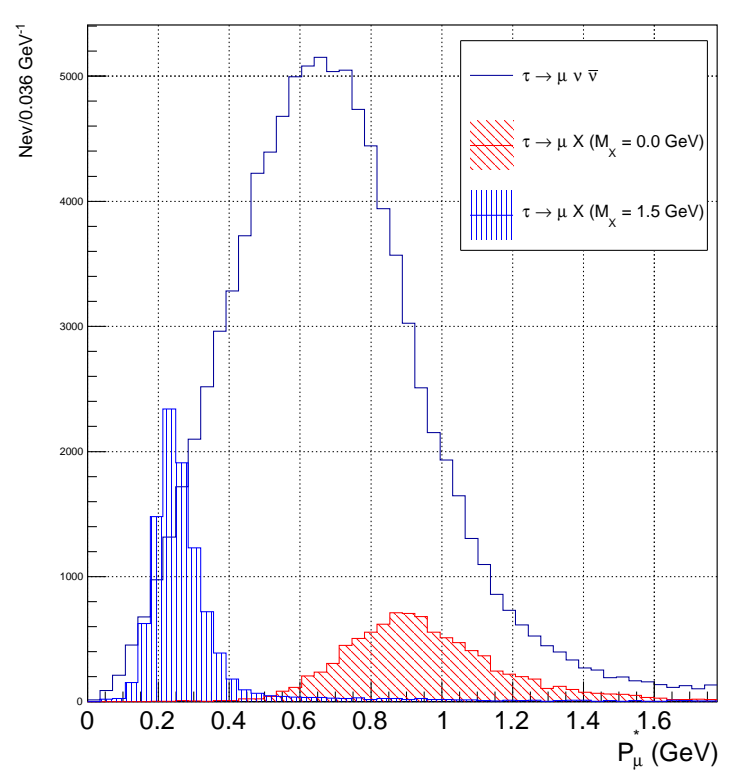

Figure 6: Distribution of calculated momentum of muon in tau rest frame $P_{\mu}^{*}$. Empty histogram shows the SM distribution of muon from $\tau \rightarrow \mu v \bar{v}$ decay. Color filled histograms represent $\tau \rightarrow \mu X$ decays on the assumption of $\mathscr{B}(\tau \rightarrow \mu X) / \mathscr{B}(\tau \rightarrow \mu v \bar{v})=10 \%$ : (blue) $m_{X}=1.5 \mathrm{GeV}$ and (red) $m_{X}=0.0 \mathrm{GeV}$. As ARGUS adopted direction of tau is approximated by that of hadron. 
Table 4: Theoretical estimates of the branching ratio of the $\tau \rightarrow \eta \pi v$ decay [15]

\begin{tabular}{|c|c|c|c|}
\hline $\mathscr{B}$ vector $\left(10^{-5}\right)$ & $\mathscr{B}$ scalar $\left(10^{-5}\right)$ & $\mathscr{B}$ vector+scalar $\left(10^{-5}\right)$ & Ref. \\
\hline 0.13 & 0.20 & 0.33 & 《15 \\
\hline 0.25 & 1.60 & 1.85 & [16 \\
\hline 0.12 & 1.38 & 1.50 & 17 \\
\hline 0.15 & 1.06 & 1.21 & [18] \\
\hline 0.36 & 1.00 & 1.36 & 19] \\
\hline $0.2-0.6$ & $0.2-2.3$ & $0.4-2.9$ & 20 \\
\hline 0.44 & 0.04 & 0.48 & 21 \\
\hline
\end{tabular}

\section{4. rare decays}

\subsection{G parity violating decay}

The G-parity operator is defined as combination of charge conjugation and the isospin as $\hat{G}=$ $\hat{C} e^{i \pi \hat{I}_{2}}$. In analogy with the ordinary spin, this operator satisfies equation $\hat{G}\left|I, I_{3}\right\rangle=\hat{C}(-1)^{I}\left|I,-I_{3}\right\rangle=$ $(-1)^{I}\left|I, I_{3}\right\rangle$, hence pion and eta have eigenvalues -1 and 1 respectively. This is one of the symmetries of the strong interaction. Therefore, if the difference of the $\mathrm{d}$ - and $\mathrm{u}$-quark masses is neglected, G-value exactly conserves. That is the reason why the current which violates G-parity is called second class. For example, $\tau \rightarrow \eta \pi v$ is typical example of G-parity-violating decay where depending on the spin of intermediate particle, the scalar and vector contributions could proceed this decay. The recent theoretical calculations of the branching ratio of $\tau \rightarrow \eta \pi v$ decays are given in Table 4 . As can be seen, various theories predict of order of $10^{-5}$.

Using $660 \mathrm{fb}^{-1}$ accumulated $e^{+} e^{-}$collision data, we searched for $\tau \rightarrow \eta \pi v$ decay where $\eta$ successively decays into $\eta \rightarrow \pi \pi \pi^{0}[22$. Figure 7 shows distribution of the invariant mass of three pions. The major contributions for the combinatorial backgrounds are $\tau \rightarrow \pi \pi \pi \pi^{0} v$ and $\tau \rightarrow \pi \pi \pi \nu$ decays. The peaking backgrounds were separately evaluated using all known decay modes which contain eta meson like $\tau \rightarrow \pi \pi^{0} \eta v, \tau \rightarrow K^{*} \eta v$ and $\tau \rightarrow K \eta v$. These expected number of background and the observed events are evaluated using Gaussian distribution. The estimated number of the signal events were $190.9 \pm 68.6$ and corresponding upper limit from null assumption became $\mathscr{B}(\tau \rightarrow \eta \pi v)<7.3 \times 10^{-5}$ at $90 \%$ confidence level.

\section{$4.2 \tau \rightarrow l v \bar{v} \gamma$ decay}

Study of the branching ratio of $\tau \rightarrow l v \bar{v} \gamma(l=e$ or $\mu)$ decays were renewed in 2015 both for experiment and theory. BaBar experiment reported the measurement using $431 \mathrm{fb}^{-1} e^{+} e^{-}$collision data by PEP-II storage ring and obtained these ratios as [23]:

$$
\begin{aligned}
& \mathscr{B}^{\mathrm{EX} .}(\tau \rightarrow e v \bar{v} \gamma)=(1.847 \pm 0.015 \pm 0.052) \times 10^{-2}, \\
& \mathscr{B}^{\mathrm{EX} .}(\tau \rightarrow \mu \nu \bar{v} \gamma)=(3.69 \pm 0.03 \pm 0.10) \times 10^{-3},
\end{aligned}
$$

where first errors are statistical and second are systematical ones. The radiative decay is defined using gamma energy threshold in tau rest frame as $E_{\gamma}^{*} \geq 10 \mathrm{MeV}$. 


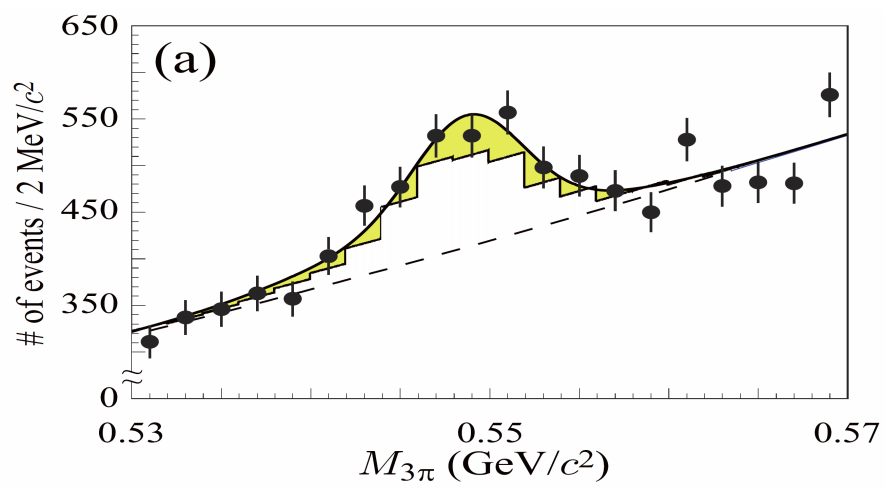

Figure 7: Distribution of invariant mass of three pions. The full and dashed lines represent fit result and combinatorial backgrounds. The histogram indicates contribution of the peaking backgrounds.

On the other side, in the renewal of theory, the next leading order QED correction was also taken into account, where up to order $\alpha^{2}$ effects were included in the calculation. The additional $\alpha$ correction gives not only loop correction, but also the infrared divergence in the final result. Therefore, a conceptual treatment of two gammas is necessary: a combination of one visible gamma and one invisible gamma $\left(\gamma_{\text {soft }}, \gamma_{\text {vis. }}\right)$ is categorized as exclusive mode while a combination where at least one visible gamma exists $\left(\gamma_{\text {vis. }}, \gamma_{\text {vis. }}\right)+\left(\gamma_{\text {soft }}, \gamma_{\text {vis. }}\right)$, is categorized as inclusive mode (both visible mode $\left(\gamma_{\text {vis. }}, \gamma_{\text {vis. }}\right)$ is also distinguished as double emission). Interestingly, the measurement of $\mathrm{BaBar}$ for $\tau \rightarrow e v \bar{v} \gamma$ decay, which is in fact the exclusive mode, disagrees with the SM prediction by $3.5 \sigma$ deviations [24]:

$$
\begin{aligned}
& \mathscr{B}^{\mathrm{Th} .}(\tau \rightarrow e v \bar{v} \gamma)=1.645(19) \times 10^{-2}, \\
& \mathscr{B}^{\mathrm{Th} .}(\tau \rightarrow \mu \nu \bar{v} \gamma)=3.572(3) \times 10^{-3} .
\end{aligned}
$$

Based on these facts, we are trying to measure the Michel Parameters using $\tau \rightarrow l v \bar{v} \gamma$ as well as $\tau \rightarrow l v \bar{v}$ decays. These parameters represent general kinematic feature of the leptonic decay and characterize the tendency of the directions and momenta of outgoing particles. From ordinary leptonic decay, $\eta, \rho, \xi$ and $\xi \delta$ are extracted [25] while the radiation in the final state additionally gives $\bar{\eta}, \xi \kappa$ and $\eta^{\prime \prime}$ 26] [27]. The deviation of these measurement from the SM prediction tells us hints of the NP.

\section{$4.3 \tau \rightarrow l l l v \bar{v}$ decay}

In the SM, the five-body leptonic decays $\tau \rightarrow l l l v \bar{v}$ occurs through an additional virtual photon to the ordinary leptonic decay $\tau \rightarrow l v \bar{v}$ and they are suppressed by order of $\alpha^{2}$. The theoretical calculation predicts these branching ratios as [28]:

$$
\begin{gathered}
\mathscr{B}^{\mathrm{Th} .}(\tau \rightarrow e e e v \bar{v})=(4.21 \pm 0.01) \times 10^{-5}, \\
\mathscr{B}^{\mathrm{Th} .}(\tau \rightarrow \mu e e v \bar{v})=(1.984 \pm 0.004) \times 10^{-5}, \\
\mathscr{B}^{\mathrm{Th} .}(\tau \rightarrow e \mu \mu \nu \bar{v})=(1.247 \pm 0.001) \times 10^{-7}, \\
\mathscr{B}^{\mathrm{Th} .}(\tau \rightarrow \mu \mu \mu \nu \bar{v})=(1.183 \pm 0.001) \times 10^{-7}
\end{gathered}
$$


Measurement of these branching ratios up to such small order of magnitude are one of the important test of the precision of QED as well as lepton universality. Furthermore, according to Ref. [29], the existence of heavy sterile neutrinos could enhance one of the branching ratio $\mathscr{B}(\tau \rightarrow e \mu \mu \nu \bar{v})$ up to $\sim 10^{-5}$.

CLEO experiment partially measured these branching ratio using $3.6 \mathrm{fb}^{-1}$ data collected by Cornell Electron Storage Ring as:

$$
\begin{aligned}
& \mathscr{B}^{\mathrm{EX}} .(\tau \rightarrow \text { eeev } \bar{v})=\left(2.7_{-1.1-0.4-0.3}^{+1.5+0.4+0.1}\right) \times 10^{-5}, \\
& \mathscr{B}^{\mathrm{EX} .}(\tau \rightarrow \mu e e v \bar{v})<3.2 \times 10^{-5} .
\end{aligned}
$$

In the event selection, in order to reject the electron and positron pair generated by gamma conversion $(\gamma \rightarrow e e)$ from $\pi^{0}$ of tau decay, special kinematic variable, sum of cosine, was used: $C=\sum_{i, j} \cos \theta_{i, j}=\cos \theta_{0,1}+\cos \theta_{1,2}+\cos \theta_{2,0}$ where indices were those of observed leptons.

We also started to measure these branching ratios using abundant number of tau pairs $N_{\tau \tau} \sim$ $10^{9}$. We updated the generator of these decays based on up-to-date theoretical formula and elaborated the selection criteria. Figure 8 shows obtained distribution of the sum of cosine for $\tau \rightarrow e e e v \bar{v}$ decay mode. At the preliminary selection criteria, we succeeded to achieve detection efficiency of $\varepsilon \sim 1.8 \%$ and purity $\sim 50 \%$.

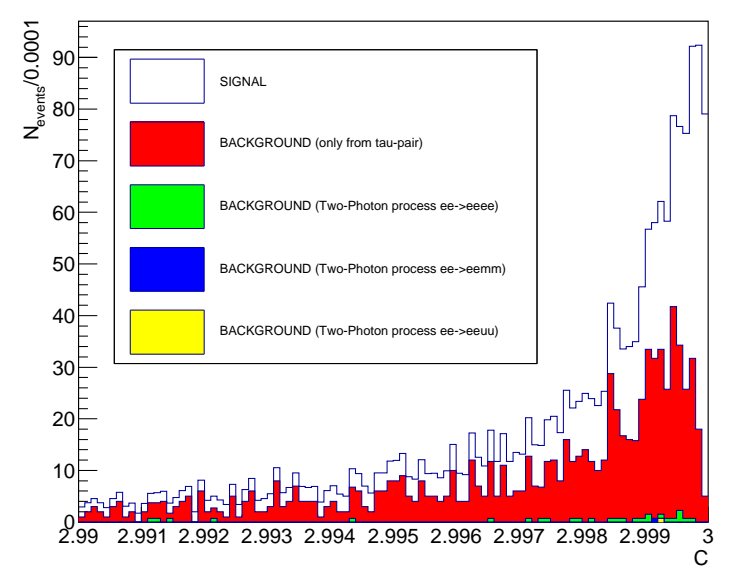

Figure 8: Distribution of sum of cosine simulated by generic MC events for $\tau \rightarrow e e e v \bar{v}$ candidates. Empty histogram represents signal (branching ratio of the SM prediction was assumed) and colored histograms show those of backgrounds: (red) from $\tau \tau$ pair, (green) $e e \rightarrow e e e e$ and (blue) $e e \rightarrow$ $e е \mu \mu$.

\section{Conclusion}

Study of the rare tau decays is one of the very attractive ways to search for the effects from New Physics. To realize the measurements of such rare decays, huge number of tau leptons is necessary and it is achieved using $B$-factory which is also known as $\tau$-factory. The KEKB accelerator delivered $\sim \mathrm{O}\left(10^{9}\right) \tau \tau$ pairs and the clean environment of $e^{+} e^{-}$collider makes it possible to do precision tests of the SM. 
Search for the LFV $\tau \rightarrow l \gamma$ and $\tau \rightarrow l l l$ decays is the most important strategy to unveil effects of New Physics, the current world best upper limits on these branching fractions, obtained at $B$-factories, are of the order of $10^{-8}-10^{-7}$. These measurements are expected to improve the sensitivities also at the next generation B-factory experiments, Belle II , with an aid of the upgrade of detector. The various LFV searches are also important to distinguish the sources of LFV decays.

Other rare decays also provide precision tests of the SM. The $\tau \rightarrow \eta \pi v$ decay is a typical mode which violates one of the symmetries of the strong interaction, G-parity. The sensitivity of Belle II is expected to reach modern theoretical prediction. Suppressed leptonic decays, $\tau \rightarrow l v \bar{v} \gamma$ and $\tau \rightarrow l l l v \bar{v}$ provide further precision tests of the electroweak mechanism.

\section{References}

[1] K.A. Olive et al., (Particle Data Group), Chin. Phys. C, 38, 090001 (2014).

[2] A. Abashian et al., Nucl. Instrum. Meth. A479 117 (2002)

[3] A. Ilakovac, A. Pilaftsis Phys. Rev. D45 1908

[4] Pham, X EPJ C8 513 (1999)

[5] G. Cvetic et al., Phys. Rev. D66 034008 (2002)

[6] Chongxing Yue et al.,Phys. Let. B547 252 (2002)

[7] T. Fukuyama et al. Phys. Rev. D68 033012 (2003)

[8] J. Ellis et al. Phys. Rev. D66 115013 (2002)

[9] A. Brignole et al., Phys. Let. B566 217 (2003)

[10] K. Hayasaka et al. Phys. Let. B666 16 (2008)

[11] K. Hayasaka et al. Phys. Let. B687 139 (2010)

[12] HFAG Tau Summer 2014 reportarXiv:1412.7515[hepex]

[13] M. Blanke et al., JHEP 0705, 013 (2007)

[14] H. Albrecht et al., Z. Phys. C68 25 (1995)

[15] S. Descotes-Genon, B. Moussallam Eur. Phys. J. C74 2946 (2014)

[16] S. Tisserant, T. Truong, Phys. Lett. B115, 264 (1982)

[17] A. Pich, Phys. Lett. B196, 561 (1987)

[18] H. Neufeld, H. Rupertsberger, Z. Phys. C68, 91 (1995)

[19] S. Nussinov, A. Soffer, Phys. Rev.D78, 033006 (2008)

[20] N. Paver, Riazuddin, Phys. Rev. D82, 057301 (2010).

[21] M. Volkov, D. Kostunin, Phys. Rev. D86, 013005 (2012).

[22] K. Hayasaka, PoS EPS-HEP 374 (2009)

[23] J. P. Lees et al. Phys. Rev. D 91051103 J. P. Lees et al.

[24] M. Fael et al., JHEP. 07. 2015153 J.

[25] D. Epifanov et al., arXiv:1409.4969 [hepex] 
[26] N. Shimizu et al., Nucl. Instrum. Meth. A824 11, 237 (2016)

[27] A. B. Arbuzov et al., arXiv:1605.06612[hepex]

[28] A. Flores-Tlalpa et al., JHEP. 04. 185 (2016)

[29] C. Dib et al., Phys. Rev. D85. 011301 (2012)

[30] M. S. Alam et al., Phys. Rev. Lett.76. 2637 (1996) 